\title{
Predictions of total and total reaction cross sections for nucleon-nucleus scattering up to $300 \mathrm{MeV}$
}

\author{
K. Amos \\ School of Physics, The University of Melbourne, Victoria 3010, Australia \\ S. Karataglidis门 \\ Theoretical Division, Los Alamos National Laboratory, Los Alamos, New Mexico, 87545 \\ P. K. Debf \\ School of Physics, The University of Melbourne, Victoria, 3010, Australia
}

(Dated: October 26, 2018)

\begin{abstract}
Total reaction cross sections are predicted for nucleons scattering from various nuclei. Projectile energies to $300 \mathrm{MeV}$ are considered. So also are mass variations of those cross sections at selected energies. All predictions have been obtained from coordinate space optical potentials formed by full folding effective two-nucleon $(N N)$ interactions with one body density matrix elements (OBDME) of the nuclear ground states. Good comparisons with data result when effective $N N$ interactions defined by medium modification of free $N N t$ matrices are used. Coupled with analyses of differential cross sections, these results are sensitive to details of the model ground states used to describe nuclei.
\end{abstract}

PACS numbers: 25.40.-h,24.10.Ht,21.60.Cs

*Electronic address: amos@physics.unimelb.edu.au

${ }^{\dagger}$ Electronic address: stevenk@lanl.gov

${ }^{\ddagger}$ Electronic address: deb@physics.unimelb.edu.au 


\section{INTRODUCTION}

Reaction cross sections from the scattering of nucleons by nuclei (stable and radioactive) are required in a number of fields of study; some being of quite current interest [1]. An example is the transmutation of long lived radioactive waste into shorter lived products using accelerator driven systems (ADS). These systems are being designed in the US, Europe, and Japan with the added objective of providing an intense neutron source to a sub-critical reactor as a new means of energy production and for which nucleon-nucleus reaction cross sections are required as well. The technology takes advantage of spallation reactions [2] within a thick high- $Z$ target (such as $\mathrm{Pb}$ or $\mathrm{Bi}$ ), where an intermediate energy proton beam induces nuclear reactions. The secondary nuclear products, particularly lower energy neutrons and protons [3], in turn induce further nuclear reactions in a cascade process. The total reaction cross sections of nucleon-nucleus scattering plays a particularly important role since the secondary particle production cross sections are directly proportional to them. Also they are inputs to intra-nuclear cascade simulations that guide ADS design. As well, nucleon-nucleus $(N A)$ cross section values at energies to $300 \mathrm{MeV}$ or more are needed not only to specify important quantities of relevance to proton and neutron radiation therapy [4], but also as they are key information in assessing radiation protection for patients.

In basic science, these total reaction cross sections are important ingredients to a number of problems in astrophysics, such as nucleosynthesis in the early universe and for aspects of stellar evolution, especially as the density distribution of neutrons in nuclei are far less well known than that of protons. Recently a link has been made between the neutron distribution in heavy nuclei, such as ${ }^{208} \mathrm{~Pb}$, and properties of neutron stars [5, 6] so heightening the need for analyses to ascertain the optimal neutron distributions in such nuclei. Analyses of proton elastic scattering angular distributions particularly for $200 \mathrm{MeV}$ protons seem to be one such method [7]. Considering the integral observables of both proton and neutron scattering from a given nucleus is another and certainly it will give direct information on the neutron rms radius; a property sought in new parity-violating electron scattering experiments [5, 7].

However, most $N A$ reaction cross sections cannot be, have not been, or are unlikely to be, measured. Thus a reliable method for their prediction is required. The usual vehicle for specifying these $N A$ total reaction cross sections has been the $N A$ optical potential; a potential most commonly taken as a local parametrized function, usually of Woods-Saxon (WS) type. However, it has long been known that the optical potential must be nonlocal and markedly so, although it has been assumed also that such nonlocality can be accounted by the energy dependence of the customary (phenomenological) models [8]. Of more concern is that the phenomenological approach is not truly predictive. The parameter values chosen, while they may be set from a global survey of data analyses, are subject to considerable uncertainties and ambiguities. This is especially true for the optical potentials for nucleon scattering from $0 p$-shell nuclei where no such global approach is valid.

We consider a predictive theory of $N A$ scattering to be one that is "direct" in that all quantities required are defined a priori. Thus each result must come from just one run of relevant codes, and there should be no post evaluation adjustments save for allowance of known a priori uncertainties in the specification of the input information.

With the nucleus viewed as a system of $A$ nucleons, $N A$ scattering is determined to first order by an optical potential formed by folding, with a suitable specification of the ground state density of the target, appropriate interactions of that projectile with each and every nucleon within the nucleus. Over the past decade or more, such microscopic approaches 
defining the $N A$ optical potential have been quite successful in predicting elastic scattering data (differential cross sections in particular). Studies forming the optical potential in both momentum and coordinate space have been made with success [8]. In the coordinate space approach, and for analyses that are based upon the DWBA programs of Raynal [9, 10], the projectile-target nucleon interaction takes the form of a complex, energy and density dependent, effective $N N$ interaction. Of those programs, DWBA98 has been used to evaluate all of the cross sections shown later herein. Appropriate effective interactions can and have been defined that, upon folding with good structure wave functions of nuclei, give credible optical potentials. Using those optical potentials, differential cross sections and spin observables such as the analyzing powers for proton scattering at many energies in the range 40 to $800 \mathrm{MeV}$ (65 and $200 \mathrm{MeV}$ in particular) and from diverse targets ranging from ${ }^{3} \mathrm{He}$ to ${ }^{238} \mathrm{U}$ have been predicted and found to have excellent agreement with data. Moreover, and very recently [7], it has been shown that analyses of differential cross sections of proton elastic scattering can select between alternative model predictions of the neutron rms radius in ${ }^{208} \mathrm{~Pb}$; such are sensitive also to the surface distribution of its neutron matter.

The microscopically formed optical potentials are complex and energy dependent from the like properties of the effective $N N$ interaction [8]. Such properties arise from mapping the effective interactions to $N N g$ matrices that are solutions of the Bruckner-Bethe-Goldstone (BBG) equations for nuclear matter. The BBG equations carry medium modification of the $N N$ scattering due to Pauli blocking and to a background mean field. Details of the effective interactions, of the folding process that gives the (nonlocal) optical potential, and of the successful predictions found therefrom of differential cross sections and analyzing powers from the scattering of protons at diverse energies and from diverse targets, are given in the literature [8].

\section{ELEMENTS OF THE OPTICAL POTENTIAL FOR NUCLEON SCATTERING}

Formally, the nonlocal optical potentials from a first order folding model can be written

$$
\begin{aligned}
U\left(\boldsymbol{r}_{1}, \boldsymbol{r}_{2} ; E\right) & =\sum_{n} \zeta_{n}\left\{\delta\left(\boldsymbol{r}_{1}-\boldsymbol{r}_{2}\right) \int \varphi_{n}^{*}(\boldsymbol{s}) v_{D}\left(\boldsymbol{r}_{1 s}\right) \varphi_{n}(\boldsymbol{s}) d \boldsymbol{s}+\varphi_{n}^{*}\left(\boldsymbol{r}_{1}\right) v_{E x}\left(\boldsymbol{r}_{12}\right) \varphi_{n}\left(\boldsymbol{r}_{2}\right)\right\} \\
& \Rightarrow U_{D}\left(\boldsymbol{r}_{1} ; E\right) \delta\left(\boldsymbol{r}_{1}-\boldsymbol{r}_{2}\right)+U_{E x}\left(\boldsymbol{r}_{1}, \boldsymbol{r}_{2} ; E\right)
\end{aligned}
$$

where $v_{D}, v_{E x}$ are combinations of the components of the effective $N N$ interactions, $\zeta_{n}$ are ground state nucleon shell occupancies (more generally they are the ground state OBDME), and $\varphi_{n}(\boldsymbol{x})$ are nucleon bound state wave functions; denoted SP functions hereafter. All details and the prescription of solution of the associated nonlocal Schrödinger equations are given in the review 8 .

The results to be discussed have been found by solving the actual nonlocal Schrödinger equations defined with potentials as given (formally) by Eq. (1). Two formulations of those optical potentials have been used. They and the results are identified by the appellations, $g$ - and $t$-folding, according that the effective $N N$ interactions have been defined by their mapping to the BBG $g$ matrices or to the basic free $N N$ scattering $t$ matrices respectively. The latter are solutions of Lippmann-Schwinger (LS) equations. In both cases, the driving $N N$ interaction has been the Bonn-B $N N$ potential [11].

From practical necessity the model descriptions of nuclei in the mass range 3 to 238 vary in complexity. With the light mass nuclei $(A \leq 12$ for example), quite large and complete 
shell model spaces with potentials either fitted or formed as $G$-matrix elements have been made [12, 13, 14]. While large space model studies of heavier nuclei are being sought, the dimensions of the problem preclude our use of all but $0 \hbar \omega$ shell model specifications for most heavier nuclei. Indeed, for targets heavier than mass 90 we have used an even simpler, packed shell, definition of their ground states. Nevertheless with such model prescriptions and using harmonic oscillator (HO) SP functions with oscillator energies selected according to an $A^{-\frac{1}{3}}$ rule, very good predictions of the scattering of 65 and $200 \mathrm{MeV}$ protons have been obtained for all but the light mass nuclei [8]. However, ${ }^{208} \mathrm{~Pb}$ and ${ }^{40} \mathrm{Ca}$ are special cases. Recently [5, 7], a Skyrme-Hartree-Fock (SHF) model of those nuclei was made and OBDME required in our folding procedure were extracted. The associated density distributions vary noticeably from that given by the HO (packed) shell model and, not surprisingly, so do proton differential cross sections.

For nuclei with $A \leq 12$ typically, better spectroscopy is needed. So also are more realistic matter distributions for studies with light mass exotic nuclei, such as of radioactive beam scattering from hydrogen. For example, the reaction cross section for $40.9 \mathrm{~A} \mathrm{MeV}{ }^{6} \mathrm{He}$ scattering from hydrogen [15 varies from $350 \mathrm{mb}$, found when ${ }^{6} \mathrm{He}$ has a neutron skin as expected with a standard shell model description, to $406 \mathrm{mb}$ when that distribution is extended further to be classified as a halo by choosing valence neutron SP functions consistent with the single neutron separation energy in ${ }^{6} \mathrm{He}$. The measured value is $409 \pm$ $21 \mathrm{mb}[15,16]$.

\section{PHASE SHIFTS, S-MATRICES, AND OBSERVABLES}

Irrespective of the means used to define $N A$ optical potentials, the objective is to define the $S$ matrix, or equivalently the (complex) phase shifts $\delta_{l}^{ \pm}(k)$, where the superscripts identify the values $j=l \pm 1 / 2$. These relate by

$$
S_{l}^{ \pm}(k)=e^{2 i \delta_{l}^{ \pm}(k)}=\eta_{l}^{ \pm}(k) e^{2 i \Re\left[\delta_{l}^{ \pm}(k)\right]}
$$

where

$$
\eta_{l}^{ \pm}(k)=\left|S_{l}^{ \pm}(k)\right|=e^{-2 \Im\left[\delta_{l}^{ \pm}(k)\right]} .
$$

With $E \propto k^{2}$, the elastic, reaction (absorption), and total cross sections respectively then are given by

$$
\begin{aligned}
\sigma_{\mathrm{el}}(E) & =\frac{\pi}{k^{2}} \sum_{l=0}^{\infty}\left\{(l+1)\left|S_{l}^{+}(k)-1\right|^{2}+l\left|S_{l}^{-}(k)-1\right|^{2}\right\}, \\
\sigma_{\mathrm{R}}(E) & =\frac{\pi}{k^{2}} \sum_{l=0}^{\infty}\left\{(l+1)\left[1-\eta_{l}^{+}(k)^{2}\right]+l\left[1-\eta_{l}^{-}(k)^{2}\right]\right\}
\end{aligned}
$$

and

$$
\begin{aligned}
\sigma_{\mathrm{TOT}}(E) & =\sigma_{\mathrm{el}}(E)+\sigma_{\mathrm{R}}(E) \\
& =\frac{2 \pi}{k^{2}} \sum_{l=0}^{\infty}\left\{(l+1)\left[1-\eta_{l}^{+}(k) \cos \left(2 \Re\left[\delta_{l}^{+}(k)\right]\right)\right]+l\left[1-\eta_{l}^{-}(k) \cos \left(2 \Re\left[\delta_{l}^{-}(k)\right]\right)\right]\right\} .
\end{aligned}
$$


The scattering amplitudes are then $2 \times 2$ matrices in the nucleon spin space and have the form,

$$
f(\theta)=g(\theta)+h(\theta) \boldsymbol{\sigma} \cdot \hat{\boldsymbol{n}}
$$

where

$$
\begin{aligned}
g(\theta) & =\frac{1}{k} \sum_{l=0}\left\{(l+1)\left[S_{l}^{+}(k)-1\right]+l\left[S_{l}^{-}(k)-1\right]\right\} P_{l}(\theta), \\
h(\theta) & =\frac{1}{i k} \sum_{l=1}\left[S_{l}^{+}(k)-S_{l}^{-}(k)\right] P_{l}^{1}(\theta) .
\end{aligned}
$$

In terms of these (complex) amplitudes, the (elastic scattering) differential cross section is defined by

$$
\frac{d \sigma}{d \Omega}=|g(\theta)|^{2}+|h(\theta)|^{2}
$$

and the analyzing power $A_{y}(\theta)$ by

$$
A_{y}(\theta)=\frac{2 \Re\left[g^{*}(\theta) h(\theta)\right]}{d \sigma / d \Omega}
$$

\section{RESULTS OF CALCULATIONS}

All results we show have been evaluated using the DWBA98 program [10], input to which are density dependent and complex effective $N N$ interactions having central, two-nucleon tensor, and two-nucleon spin-orbit components. The effective interactions we use have been generated for energies from $10 \mathrm{MeV}$ to over $300 \mathrm{MeV}$ in $10 \mathrm{MeV}$ steps by an accurate mapping to $N N$ t- and $g$ matrices found by solutions of the LS and BBG equations respectively and based usually upon the Bonn $N N$ potentials. Details are given in the review [8].

Other input to DWBA98 are the ground state occupancies (or OBDME) and the associated SP functions. The SP functions used in most of the calculations for nuclei of mass 20 and above at best come from a $0 \hbar \omega$ shell model which has been adopted to describe their ground state occupancies. For the lighter mass nuclei considered, larger shell model spaces

were used to define their ground states, and in some cases the interaction potentials defined as $G$ matrix elements of a realistic interaction [12]. In shell model studies using those $G$ matrix elements, the oscillator energy $(\hbar \omega)$ for the SP functions also is specified. As stated earlier, the cases of ${ }^{208} \mathrm{~Pb}$ and ${ }^{40} \mathrm{Ca}$ are special in that we have used structure information taken from recent SHF studies [5, 0].

\section{A. Energy variation of proton total reaction cross sections}

In this subsection we present our predictions of the total reaction cross sections for proton scattering up to $300 \mathrm{MeV}$ for diverse nuclei, ranging in mass from ${ }^{6} \mathrm{Li}$ to ${ }^{238} \mathrm{U}$. In all cases, at least two calculations were made. The first of these used the effective interaction defined from the $t$ matrices of the Bonn-B interaction while with the second, that built upon the 
TABLE I: Data source table for proton and neutron reaction data used

\begin{tabular}{|c|c|}
\hline Nucleus & Proton references (in year order) \\
\hline${ }^{6} \mathrm{Li}$ & [19], 20] \\
\hline${ }^{9} \mathrm{Be}$ & [21], [19], [22], 23], [24], [25], [26], [27], [28] \\
\hline${ }^{12} \mathrm{C}$ & [21], [29], [30], [31], [32], [19], [33], [34], [35], [23], [36], 24], [26], [27], 28] \\
\hline${ }^{16} \mathrm{O}$ & [37], [24, [20] \\
\hline${ }^{19} \mathrm{~F}$ & [37, 20] \\
\hline${ }^{27} \mathrm{Al}$ & [21], [29], [31], 32], 38], [19], 33], [22], [35], 39], 440, 23], 36], [24], 26] \\
\hline${ }^{40} \mathrm{Ca}$ & [19], [41], [23], [42], [20], [28] \\
\hline${ }^{63} \mathrm{Cu}$ & [29], [43], [33], [22], [44], [35], [39], 440], [23], 445], 446], 24] \\
\hline${ }^{90} \mathrm{Zr}$ & [22], 23], 45], 36] \\
\hline${ }^{118} \mathrm{Sn}$ & [31], [32], [22], 23], 445], [24], 447, [28] \\
\hline${ }^{140} \mathrm{Ce}$ & 48 \\
\hline${ }^{159} \mathrm{~Tb}$ & 23], 49 \\
\hline${ }^{181} \mathrm{Ta}$ & [22], [23, 49] \\
\hline${ }^{197} \mathrm{Au}$ & [19], [22], [35], [39], [23], 446], 449] \\
\hline${ }^{208} \mathrm{~Pb}$ & [29], 31], [32], 33], 41], [40], 23], 36], [24], 25], 20], 28] \\
\hline${ }^{238} \mathrm{U}$ & 21], 23] \\
\hline
\end{tabular}

associated $g$ matrices was used. Comparison of the results of each pair of calculations demonstrates the effects in predictions due to the medium modification to the free NN interaction that define the $g$ matrices. The ensuing $t$ - and $g$-folding results are portrayed in the figures by the dashed and solid curves respectively.

As noted, the structure models of the light mass nuclei involve diverse complete $N \hbar \omega$ bases. For the lightest, ${ }^{6} \mathrm{Li}$, a complete $(0+2+4) \hbar \omega$ model of structure has been used, while for ${ }^{9} \mathrm{Be}$ and ${ }^{12} \mathrm{C}$ the OBDME have been defined from complete $(0+2) \hbar \omega$ shell model calculations [17]. In addition we have calculated the reaction cross sections from ${ }^{118} \mathrm{Sn}$ and ${ }^{159} \mathrm{~Tb}$ allowing the outer (neutron) shell to have a smaller (15-20\%) harmonic oscillator energy. By that means, the neutron surface of each is slightly more extended than with the base (packed shell) model forms; a very simple allowance for any effect of ground state deformations. This idea for varied surface SP functions within an HO model has been used in the guise of a two frequency shell model [18]. Our wave functions are not so well determined of course.

The results for scattering from ${ }^{6} \mathrm{Li},{ }^{9} \mathrm{Be},{ }^{12} \mathrm{C}$ and from ${ }^{16} \mathrm{O}$ are displayed respectively in segments (a), (b), (c) and (d) of Fig. 1. The experimental data shown therein were taken

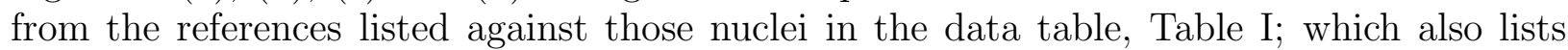
the sources of the data that are shown in the figures to follow. The data from the four lightest mass nuclei are well reproduced by $g$-folding calculations made with large space shell model structure (solid curve), but they are not with $t$-folding calculations (dashed curve). However, large space structure calculations are necessary if one is to describe the physics even adequately. As indicated above, for ${ }^{6} \mathrm{Li}$, such structure was found from a shell model calculation [50] made using a compete $(0+2+4) \hbar \omega$ space while those for ${ }^{9} \mathrm{Be},{ }^{12} \mathrm{C}$ and ${ }^{16} \mathrm{O}$ were made using complete $(0+2) \hbar \omega$ models. Results found using the simpler $0 \hbar \omega$ structure model of Cohen and Kurath [51] within $g$-folding, underestimate the data at most 


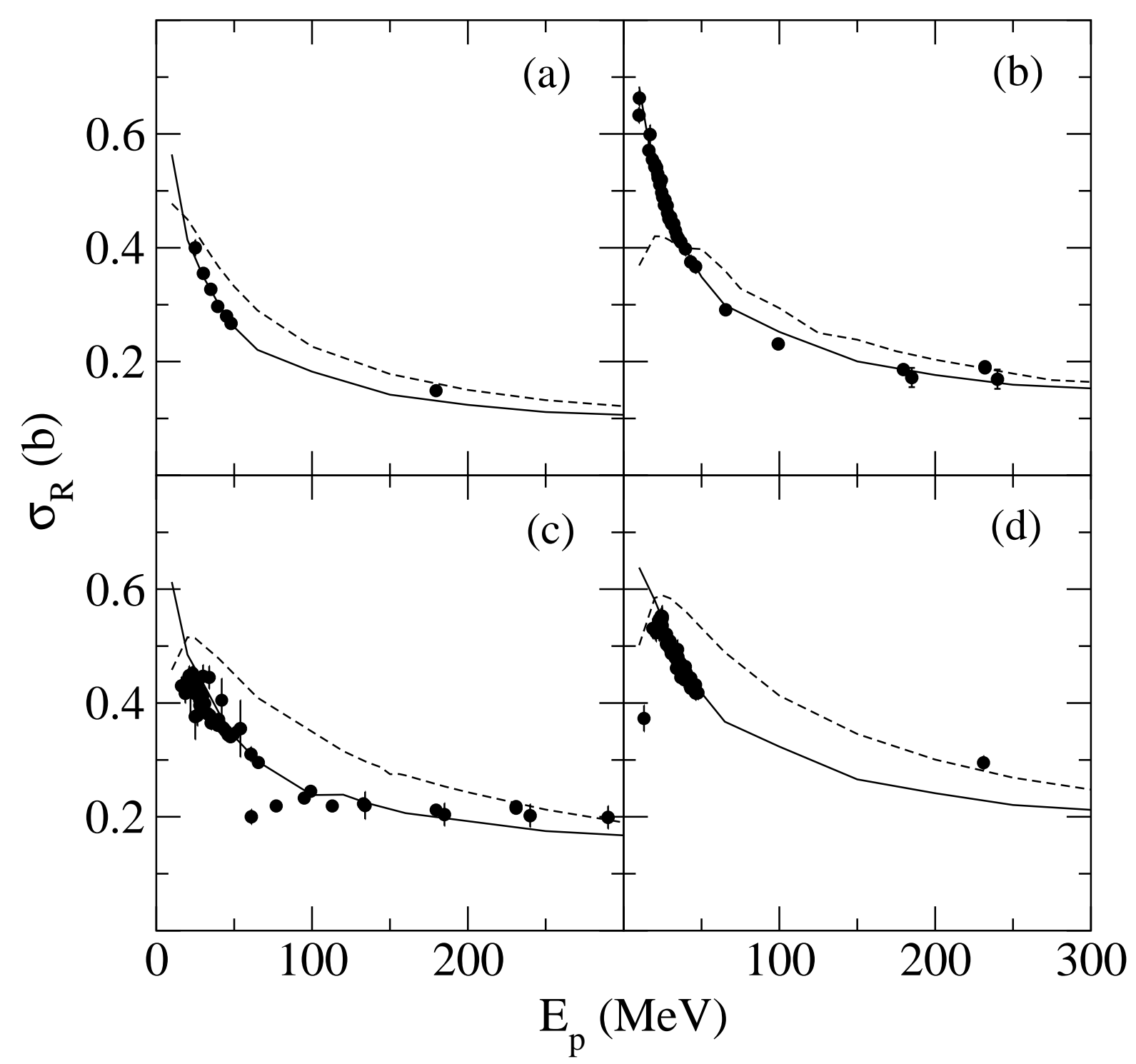

FIG. 1: Energy dependencies of $\sigma_{R}$ for proton scattering from (a) ${ }^{6} \mathrm{Li}$, (b) ${ }^{9} \mathrm{Be},(\mathrm{c}){ }^{12} \mathrm{C}$, and (d) ${ }^{16} \mathrm{O}$. The solid curves are predictions made using $g$-folding optical model calculations while those found using $t$-folding optical potentials are displayed by the dashed curves.

energies, and for ${ }^{9}$ Be particularly. This reflects the too compressed density profile for the nuclei given by the simpler model. Results are displayed for proton energies from $10 \mathrm{MeV}$. Although experimental data exist to lower energies in these cases, we do not consider the first order folding prescription for the optical potential to be appropriate in the low energy regime of scattering from these nuclei. Up to and over $20 \mathrm{MeV}$ excitation, their spectra have numerous distinguishable states. Excitations to regions of low level density are not taken into account forming the optical potentials. We have confidence in the optical potentials when the input energy coincides to excitations to regions of high level density and where particle emission is feasible.

The ${ }^{12} \mathrm{C}$ results in particular are worth comment. For this nucleus, as with the others, the reaction cross sections obtained from those $g$-folding calculations for ${ }^{12} \mathrm{C}$ are in very good agreement with the experimental data up to $300 \mathrm{MeV}$. Most evidently, the medium 


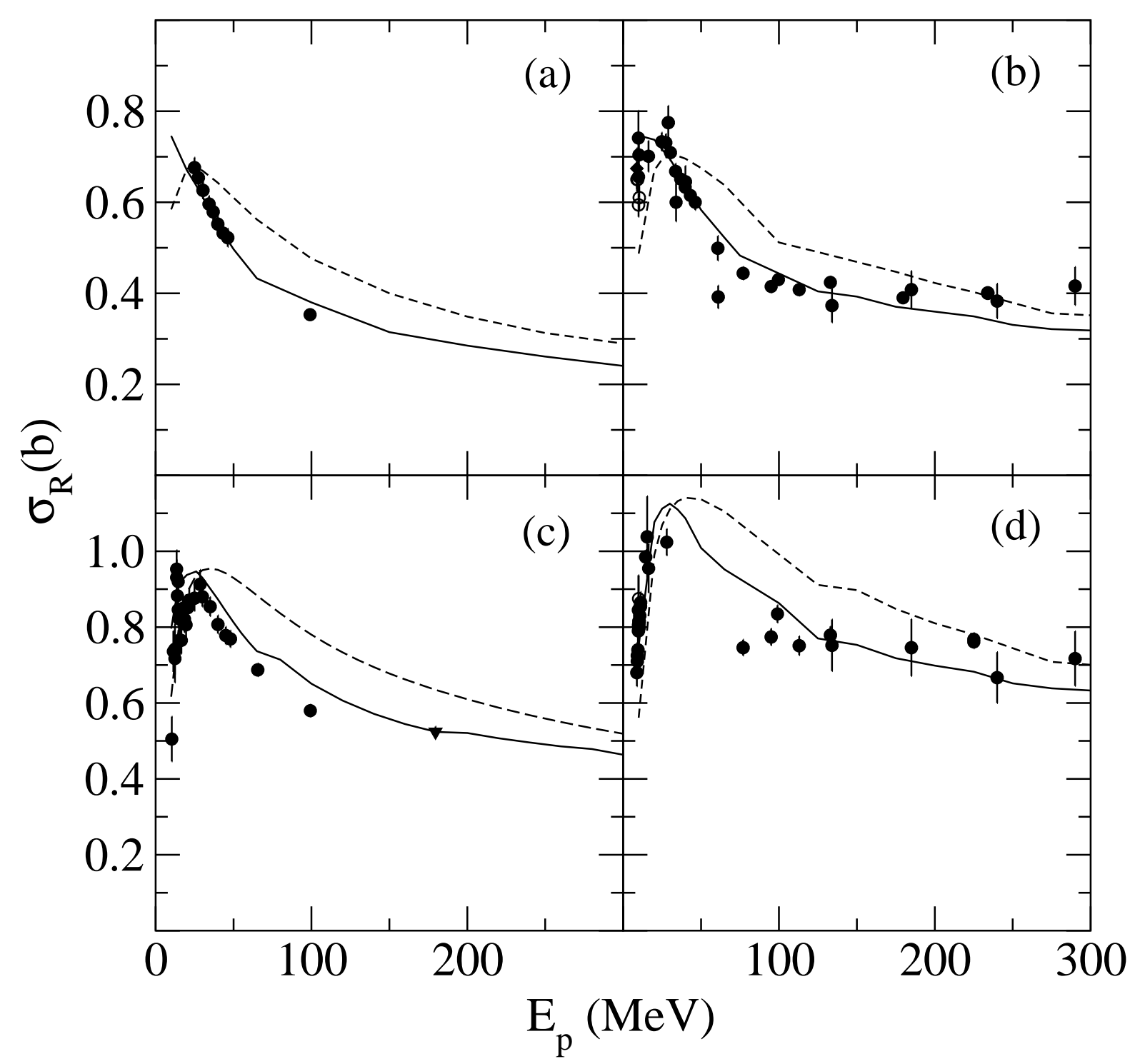

FIG. 2: Energy dependencies of $\sigma_{R}$ for proton scattering from (a) ${ }^{19} \mathrm{~F}$, (b) ${ }^{27} \mathrm{Al}$, (c) ${ }^{40} \mathrm{Ca}$, and (d) ${ }^{63} \mathrm{Cu}$. The notation is as for Fig. 1.

effects differentiating the $g$ - from the $t$ - matrices used in the folding scheme defining the optical potentials are required for predictions to match observation. The $t$-folding model overestimates the data by $20-40 \%$ within the energy regime below $200 \mathrm{MeV}$. But some data, notably at $61 \mathrm{MeV}$ [32] and at $77 \mathrm{MeV}$ [33] $\mathrm{MeV}$ with ${ }^{12} \mathrm{C}$, are in disagreement with both calculated results.

Predictions for proton scattering from ${ }^{19} \mathrm{~F},{ }^{27} \mathrm{Al},{ }^{40} \mathrm{Ca}$, and from ${ }^{63} \mathrm{Cu}$ respectively are compared with the data in the segments (a), (b), (c) and (d) of Fig. 2. (As noted above the wave functions for ${ }^{40} \mathrm{Ca}$ were obtained from an SHF model [7].) Again all data are well reproduced by the $g$-folding calculations. With ${ }^{27} \mathrm{Al}$ however, three data points between 180 and $300 \mathrm{MeV}$ are in better agreement with the results of $t$-folding calculations while one data point, at $61 \mathrm{MeV}$ [32], is in disagreement with both calculations.

Data are shown in this figure again from $10 \mathrm{MeV}$ but with ${ }^{40} \mathrm{Ca}$ in particular, the folding model approach is not expected to be reliable at the energies in the range 10 to $20 \mathrm{MeV}$. 


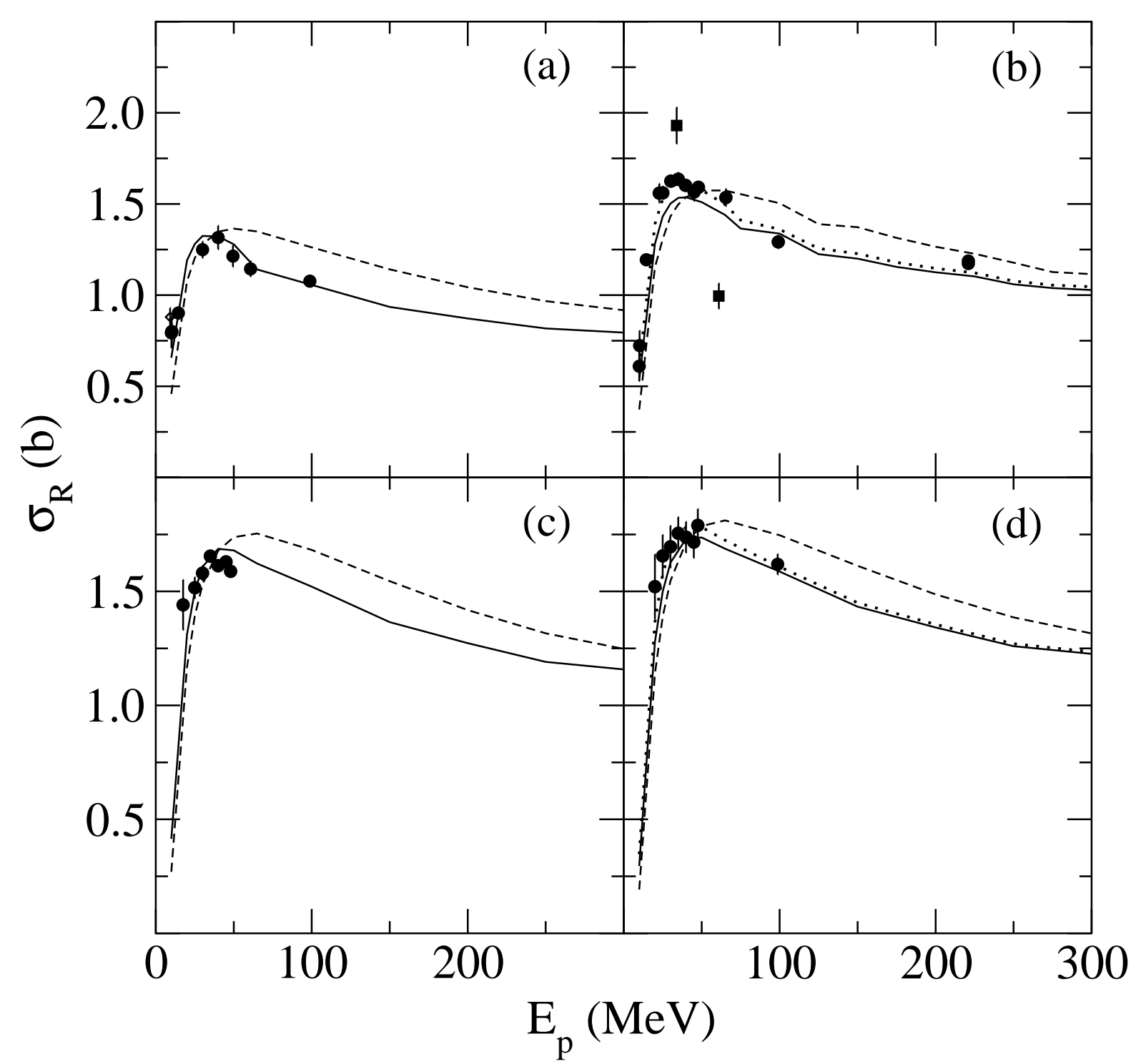

FIG. 3: Energy dependencies of $\sigma_{R}$ for proton scattering from (a) ${ }^{90} \mathrm{Zr}$, (b) ${ }^{118} \mathrm{Sn}$, (c) ${ }^{140} \mathrm{Ce}$, and (d) ${ }^{159} \mathrm{~Tb}$. Basic notation is as for Fig. 1, but now also with dotted curves that display predictions obtained with the $g$-folding model potentials formed with the outer most shell neutrons specified by a harmonic oscillator with an oscillator length $10 \%$ larger.

Those excitations energies correspond to a region of low level density in ${ }^{40} \mathrm{Ca}$. Indeed the reaction data from ${ }^{40} \mathrm{Ca}$ show rather sharp resonance-like features below $20 \mathrm{MeV}$. For ${ }^{63} \mathrm{Cu}$ however, no such sharp structures are evident in the reaction cross section data and our prediction with a $g$-folding potential at $10 \mathrm{MeV}$ gives a value in quite reasonable agreement with observation. With both ${ }^{40} \mathrm{Ca}$ and ${ }^{63} \mathrm{Cu}$, the $g$-folding results are in very good agreement with the data for energies above $20 \mathrm{MeV}$. That is in stark contrast to the $t$-folding results. The $t$-folding results underestimate the data below $20 \mathrm{MeV}$ and overestimate considerably the data above $40 \mathrm{MeV}$. In the 20 to $40 \mathrm{MeV}$ zone, both calculations give results in reasonable agreement. Such trends are evident for most heavy nuclei.

In Fig. 3, we present the data and our predictions of the total reaction cross sections for proton scattering from ${ }^{90} \mathrm{Zr},{ }^{118} \mathrm{Sn},{ }^{140} \mathrm{Ce}$ and ${ }^{159} \mathrm{~Tb}$. They are shown in segments (a), (b), (c), 
and (d) respectively and compared against data taken from the relevant references given in Table I. Again results from $g$-folding calculations are in very good agreement with the data while the $t$-folding results are overestimates at and above $40 \mathrm{MeV}$ and underestimates the data below $20 \mathrm{MeV}$. The third $p^{-}{ }^{118} \mathrm{Sn}$ total reaction cross section result given in segment (b) of Fig. 3 and portrayed in that figure by the dotted curves, was obtained from a $g$-folding optical potential formed by varying the surface neutron orbit $\left(h_{11 / 2}\right)$ to be that for an oscillator length increased by $10 \%$ from our basic calculation. With the (slightly) extended neutron distribution that results, the $g$-folding potential total reaction cross sections then are in very good agreement with the data; save for the ubiquitous $61 \mathrm{MeV}$ value. Likewise there is a datum at $32 \mathrm{MeV}$ at odds with our results. But that point also is at odds with other data. Also in the mismatch collection, the datum at $17.5 \mathrm{MeV} p-{ }^{140} \mathrm{Ce}$ scattering is underestimated. For ${ }^{159} \mathrm{~Tb}$, the $g$-folding result (solid curve) is still a quite good replication of data but the calculations obtained from $g$-folding optical potentials formed by varying the surface neutron orbit $\left(h_{9 / 2}\right)$ to be that for an oscillator length increased by $10 \%$ (dotted curve) are better.

In segments (a), (b), (c) and (d) of Fig. 1, we compare the calculated total reaction cross sections with proton scattering data from ${ }^{181} \mathrm{Ta},{ }^{197} \mathrm{Au},{ }^{208} \mathrm{~Pb}$, and ${ }^{238} \mathrm{U}$. Again all data are best and well described by the predictions we make with the $g$-folding model. There is a cross over regime in energy where the predictions of our $t$ - and $g$-folding potential equate. The differential cross sections do not however and when such are also considered [8], preference is given to the $g$-folding potential results at all energies.

The dotted curve in the case of ${ }^{208} \mathrm{~Pb}$ [segment (c)] results when the oscillator length for the outer neutron shell $\left(i_{13 / 2}\right)$ of the simple packed shell model we have used to describe the nucleus is increased by $8 \%$. The associated increase in the matter profile brings the predicted reaction cross sections then in very good agreement with observation. Using the SHF wave functions [5], gives the result displayed by the dot-dashed curve in this figure. Clearly using these new functions has made a slight change to the predictions found with the simple HO packed model (solid curve); the modified HO model result is in better agreement with that of the SHF model.

Exceptional data points are found at $19.8 \mathrm{MeV}$ in ${ }^{181} \mathrm{Ta}$ where that data point is underestimated with our calculation by $20 \%$, at $29 \mathrm{MeV}$ in ${ }^{197} \mathrm{Au}$, and in ${ }^{208} \mathrm{~Pb}$ near 30,61 and $77 \mathrm{MeV}$. Such exceptional points were also noted in the data from lighter mass nuclei. But in most cases, those exceptional point values do not agree with other measurements made at close values of energy. For example other data from ${ }^{208} \mathrm{~Pb}$ taken at $60.8 \mathrm{MeV}$ [36 and at $65.5 \mathrm{MeV}$ [28] give different results and in fact reaction cross section values that are consistent with our predictions. We note that Menet et al. [36 argue for a much larger systematic error in the studies reported in the relevant earlier experiments.

\section{B. Mass variation of proton total reaction cross sections}

The mass variations of total reaction cross sections for the scattering of 25, 30, 40, 65, 100 and $175 \mathrm{MeV}$ protons are shown in the different segments (as labeled) of Fig. 5. From that figure, it is evident that the $g$-folding results are in quite good agreement with data while the $t$-folding results underestimate most of the $25 \mathrm{MeV}$ data, are in reasonable agreement with the 30 and $40 \mathrm{MeV}$ data but overestimate most of the $65 \mathrm{MeV}$ data. Note that the extended matter SP states have been used with the ${ }^{208} \mathrm{~Pb}$ and the $\mathrm{Sn}$ isotopes calculations.

The disparities between the $t$ - and $g$-folding potential results for the reaction cross sections 


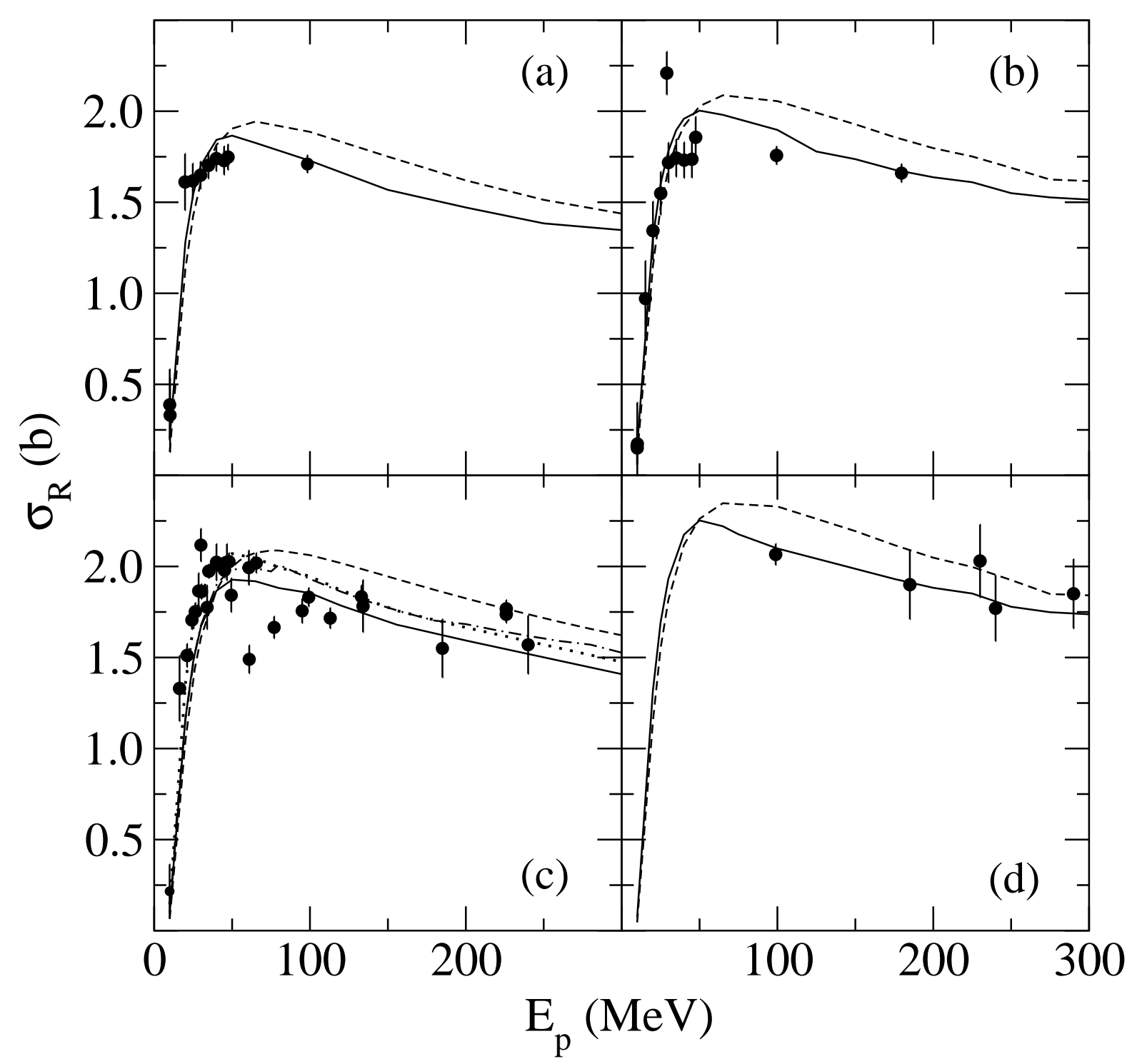

FIG. 4: Energy dependencies of $\sigma_{R}$ for proton scattering from (a) ${ }^{181} \mathrm{Ta}$, (b) ${ }^{193} \mathrm{Au}$, (c) ${ }^{208} \mathrm{~Pb}$, and (d) ${ }^{238} \mathrm{U}$. Basic notation is as for Fig. 1. For ${ }^{208} \mathrm{~Pb}$, the dotted curve is the result of extending the $i_{13 / 2}$ neutron orbit by increasing the oscillator length for that shell by $8 \%$. The dot-dashed curve is the predictions found using SHF wave functions.

are more evident at higher energies. In segments (e) and (f) of Fig. 5 we display the mass variation of the total reaction cross sections measured [52 at 100 and $175 \mathrm{MeV}$. Again, the $g$-folding model predictions are in excellent agreement with the measured values, while the $t$-folding results overestimate observations typically by $150 \mathrm{mb}$. At $100 \mathrm{MeV}$ proton scattering, proton total reaction cross sections from many nuclei in the mass range to ${ }^{238} \mathrm{U}$ have been measured and it is very clear that the $g$-folding model predictions are in good agreement with them. Fewer measurements have been made at $175 \mathrm{MeV}$, but they too span the mass range to ${ }^{238} \mathrm{U}$ and the results of those measurements also are in very good agreement with the $g$-folding optical model predictions.

We have seen in most of the previous figures that, at some energy point, the total reaction cross sections obtained by the $t$-folding and $g$-folding calculations are same. In Fig. 6 we 


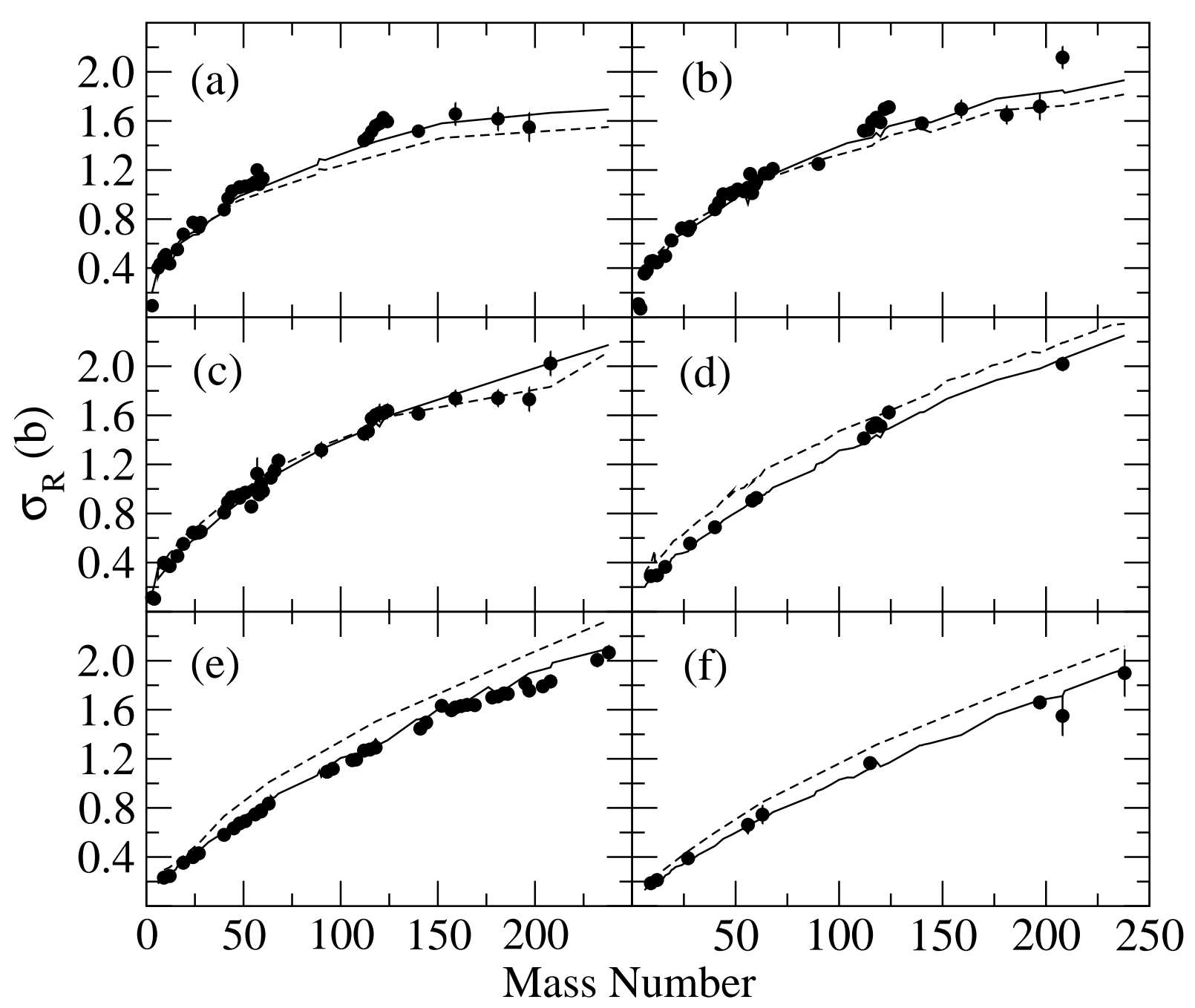

FIG. 5: The mass variations of proton reaction cross sections at diverse energies. Scattering at energies of 25, 30, 40, 65, 100, and $175 \mathrm{MeV}$ are presented in segments (a), (b), (c), (d), (e), and (f), respectively.

display those crossing energy values as a function of $A^{1 / 3}$; the energy is in MeV. It clearly indicates that there is a line in mass and energy above and below which the $g$-folding calculation results are smaller and larger respectively than the corresponding $t$-folding ones.

\section{Energy/mass variations of neutron total cross sections}

Accurate measurements of neutron total reaction cross sections are far more difficult to achieve than their proton counterparts. Indeed usually those cross sections are obtained by subtracting the elastic from the total scattering cross section. While both of those cross sections can be measured with some accuracy, the subtraction of two large numbers with attendant uncertainties is subject to numerical problems. We note, however, that a new technique from Japan for measuring neutron total reaction cross sections utilizing in-beam and out-beam methods similar to those used in proton scattering shows promise. Nevertheless, herein, we concentrate on analyses of total scattering cross section data. 


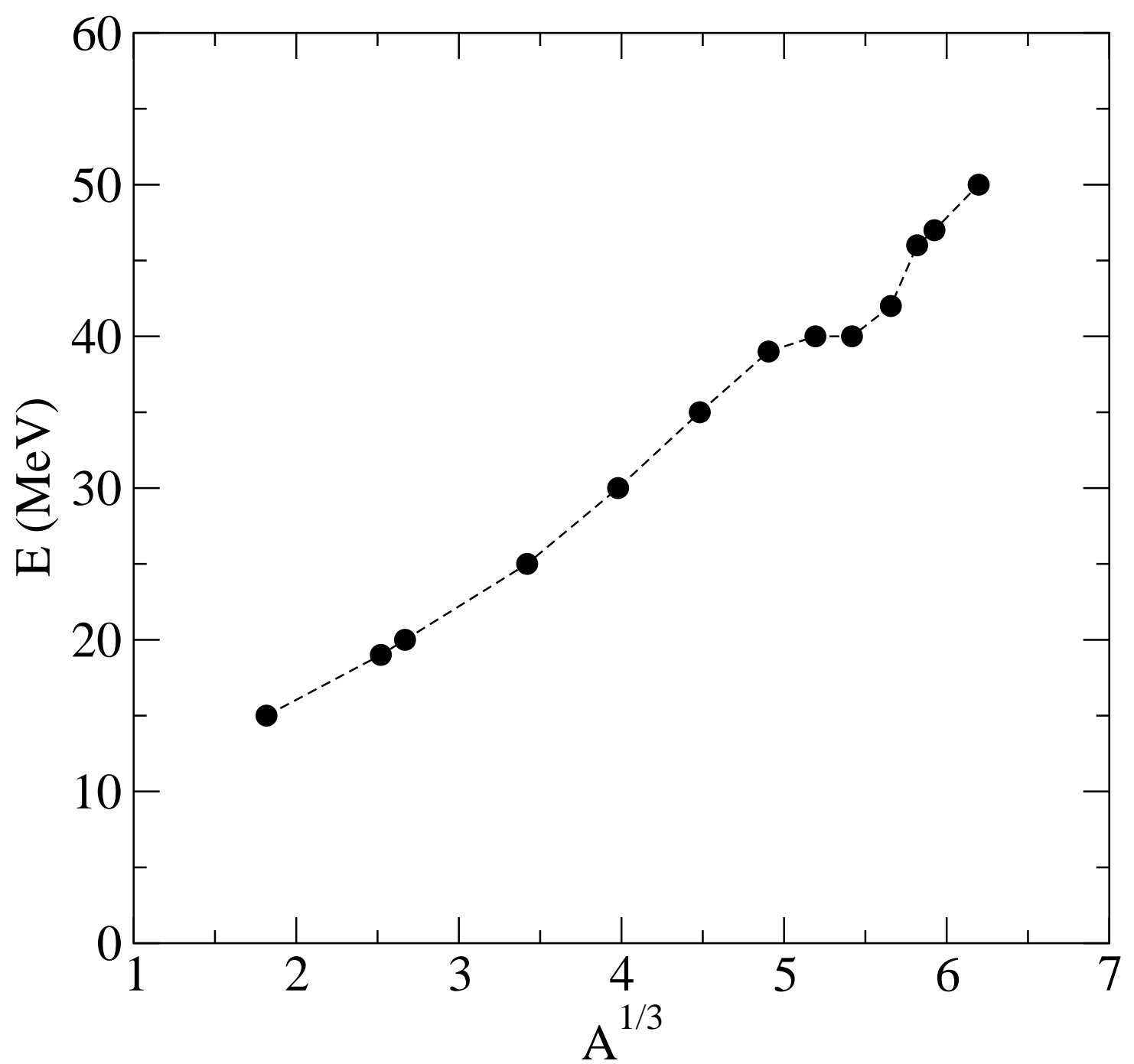

FIG. 6: The mass-energy equivalence points for $t$ - and $g$-folding results for proton total reaction cross sections. The line is drawn only to guide the eyes.

The data we have chosen to analyze have been taken from a recent survey by Abfalterer et al. [53]. That survey includes data measured at LANSCE that are supplementary and additional to those published earlier by Finlay et al. [54. From that recent data compilation, we have selected cases for study having target masses that span the stable mass range and which includes as many of the nuclei as possible for which we have analyzed proton scattering data. Specifically we calculate neutron total scattering cross sections for ${ }^{6} \mathrm{Li},{ }^{12} \mathrm{C},{ }^{19} \mathrm{~F},{ }^{40} \mathrm{Ca}$, ${ }^{89} \mathrm{Y},{ }^{184} \mathrm{~W},{ }^{197} \mathrm{Au},{ }^{208} \mathrm{~Pb}$ and ${ }^{238} \mathrm{U}$. In some cases the data taken were from natural targets; we indicate those as discussion of them occurs in the text. Given that analyses with the $g$-folding optical potential has been found the more appropriate with the proton scattering analyses, we only present in the ensuing figures, results obtained from the $g$-folding optical models for neutron scattering. In forming those neutron optical potentials, the structure models used were those that defined proton scattering. The effective $N N$ interactions were as well.

Predictions of the total cross sections for neutrons scattered from ${ }^{6} \mathrm{Li},{ }^{12} \mathrm{C},{ }^{19} \mathrm{~F}$, and ${ }^{40} \mathrm{Ca}$ 


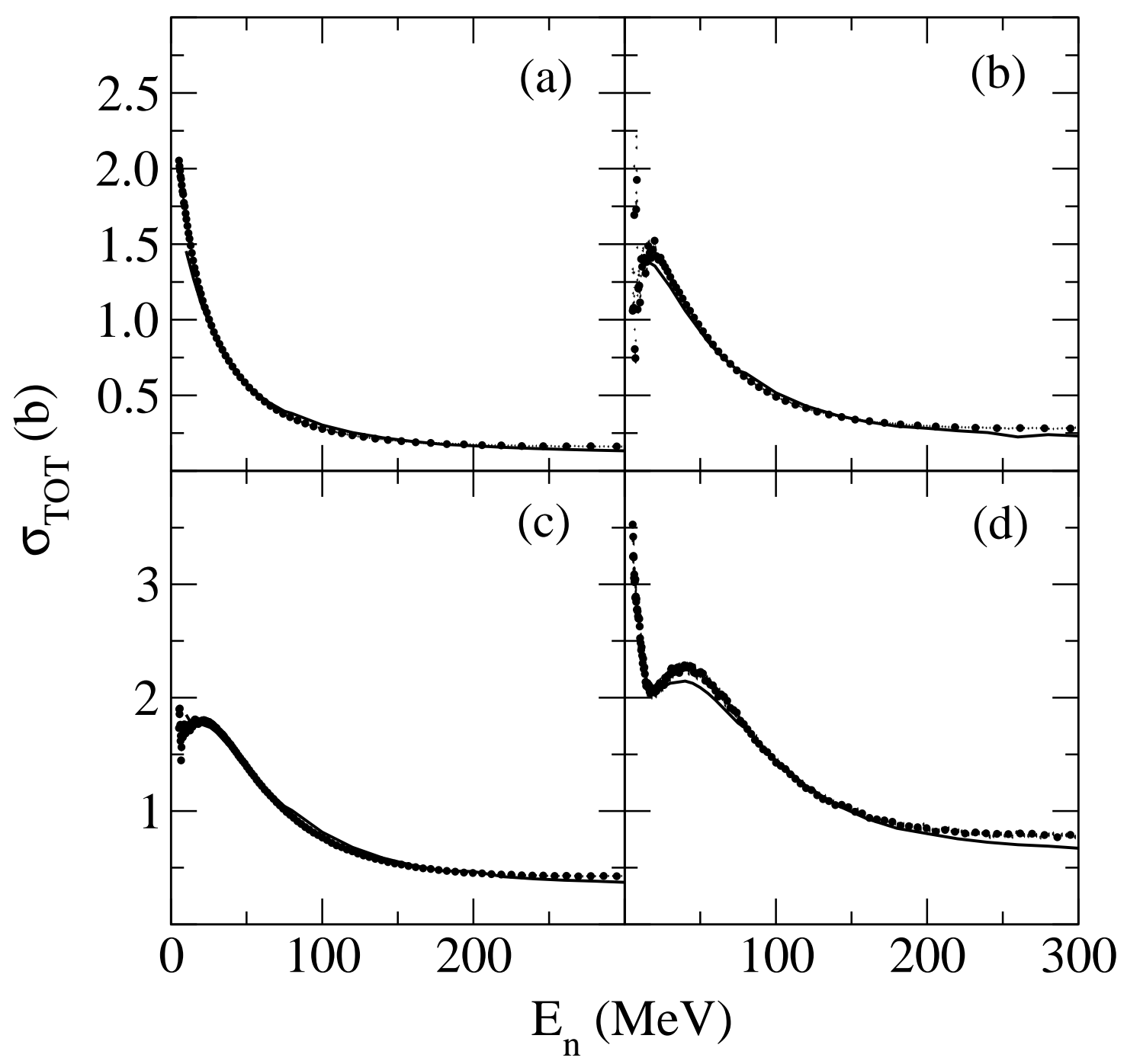

FIG. 7: Total cross sections for neutrons scattered from (a) ${ }^{6} \mathrm{Li}$, (b) ${ }^{12} \mathrm{C}$, (c) ${ }^{19} \mathrm{~F}$, and (d) ${ }^{40} \mathrm{Ca}$. The data are those of Abfalterer et al. [53]; in (b) the data correspond to scattering from natural carbon.

are compared to the data in Fig. 7. The models for ${ }^{6} \mathrm{Li},{ }^{12} \mathrm{C}$, and ${ }^{40} \mathrm{Ca}$ to specify the densities were those used in the calculations of proton scattering. The model for ${ }^{19} \mathrm{~F}$ was a $0 \hbar \omega$ shell model calculation using the WBT interaction of Warburton and Brown [55]. The oscillator parameter for the HO SP functions in that case was $1.855 \mathrm{fm}$, chosen to reproduce the rms radius of ${ }^{19} \mathrm{~F}(2.9 \mathrm{fm}[56])$. In all cases except for ${ }^{40} \mathrm{Ca}$, there is excellent agreement with the data, and the turnover in the cross sections at $\sim 20 \mathrm{MeV}$ for natural carbon and ${ }^{19} \mathrm{~F}$ are predicted. The low-energy structure in the cross section for ${ }^{40} \mathrm{Ca}$ is reproduced, although the peak at $40 \mathrm{MeV}$ is underpredicted by $10 \%$. Also for ${ }^{40} \mathrm{Ca}$ the cross section at $300 \mathrm{MeV}$ is underpredicted.

Our predictions of the total cross sections for neutrons scattering from ${ }^{89} \mathrm{Y},{ }^{184} \mathrm{~W},{ }^{197} \mathrm{Au}$, and ${ }^{238} \mathrm{U}$ are compared to the data in Fig. 8. In the case of ${ }^{184} \mathrm{~W}$, the data were taken from scattering using natural tungsten as the target. The structure of three of these nuclei was obtained from a simple packed model as used previously. For ${ }^{89} \mathrm{Y}$, however, the shell model of Ji and Wildenthal [57] was used to specify the density. The four results exhibit the same 


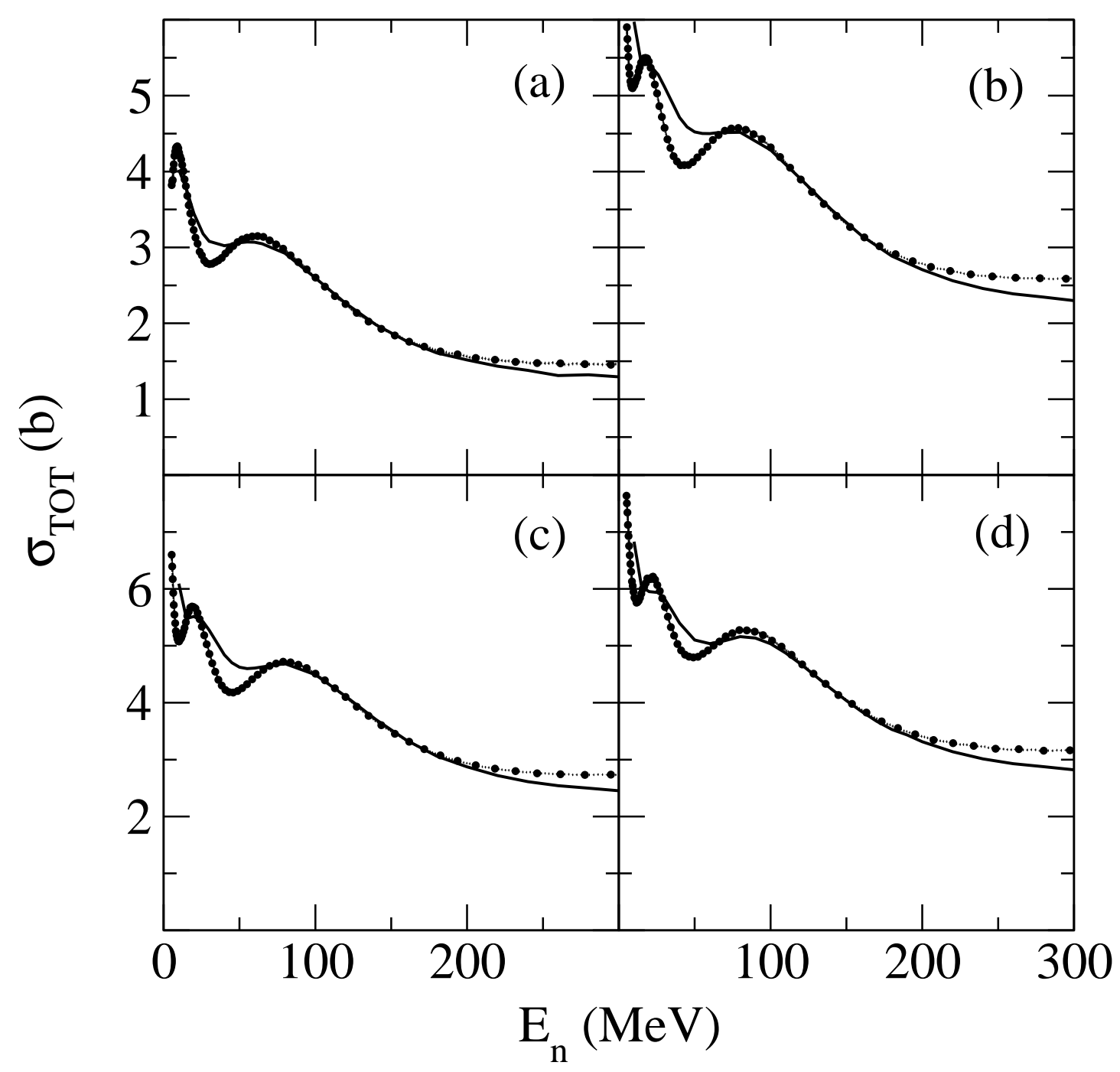

FIG. 8: Total cross sections for neutrons scattered from (a) ${ }^{89} \mathrm{Y}$, (b) ${ }^{184} \mathrm{~W}$, (c) ${ }^{197} \mathrm{Au}$, and (d) ${ }^{238} \mathrm{U}$. The data are those of Abfalterer et al. [53]; in (b) the data correspond to scattering from natural tungsten.

features. The predicted cross sections agree well with the experimental results between 70 and $200 \mathrm{MeV}$, with the peak cross section values slightly underpredicted. Below $70 \mathrm{MeV}$ the cross sections are overpredicted but the structural character exhibited in the data is found. To obtain better agreement at energies 40 to $70 \mathrm{MeV}$ at least, improvements in the SP functions are required. Improvements such as by using a SHF model seem needed, given the results we shall present next for ${ }^{208} \mathrm{~Pb}$. Above $200 \mathrm{MeV}$, all results underpredict the data. We believe that this indicates that our present effective interaction for those energies at and about the pion production threshold need be improved. Possibly more explicit contributions from the $\Delta$ resonance in the $N N$ interaction in defining the effective $N N$ force to be used in the $g$-folding giving the optical potentials are needed [58].

As stated above in the case of proton scattering, ${ }^{208} \mathrm{~Pb}$ is a special case. To specify the density of ${ }^{208} \mathrm{~Pb}$ we have used the SHF calculation of Brown [5, 7]. The results of our calculations of the total neutron scattering cross section from ${ }^{208} \mathrm{~Pb}$ using that model are 


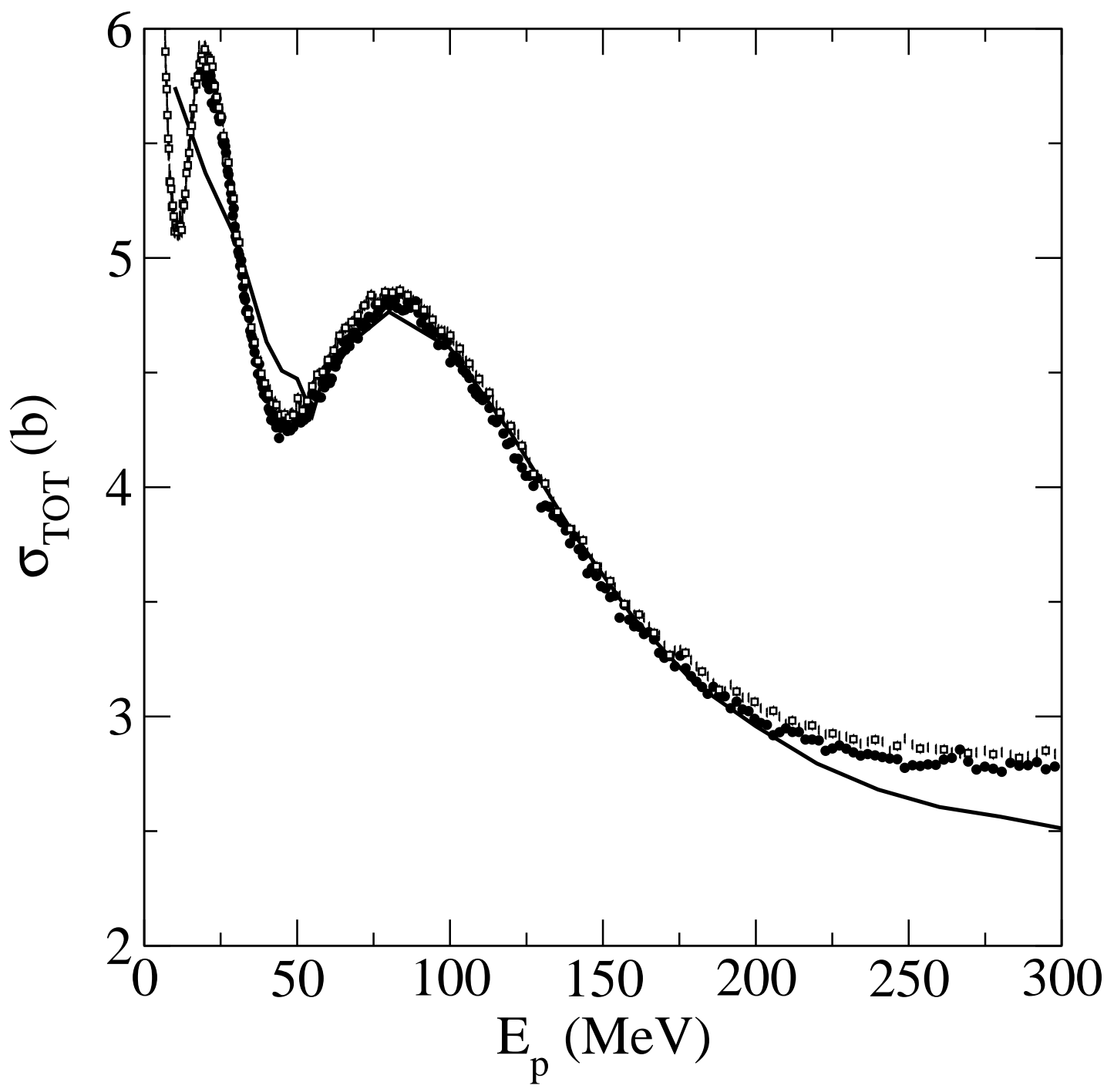

FIG. 9: Total cross section for the scattering of neutrons from ${ }^{208} \mathrm{~Pb}$. The results of our calculations are compared to the data of Finlay et al. [54] (circles) and of Abfalterer et al. [53] (squares). The latter set correspond to scattering from natural $\mathrm{Pb}$.

given in Fig. 9. Therein, we compare our prediction with the data of Finlay et al. [54] and of Abfalterer et al. [53]. The latter set correspond to scattering from natural Pb. From 60 to $200 \mathrm{MeV}$, the agreement with the data is excellent, as it was for the other nuclei also. Below $60 \mathrm{MeV}$, the energy dependence is reproduced, although the minimum at $50 \mathrm{MeV}$ is slightly overpredicted. Note that when the oscillator model is used, the calculated results do not reproduce the data in this energy regime as adequately [1]. Above $200 \mathrm{MeV}$, the predicted cross section falls too sharply as it did for the other scattering cases. The variation of the structure model from HO to SHF did not influence much the result above $200 \mathrm{MeV}$. Again we think that our effective force may be at fault for this data at these energies. The mismatch above $200 \mathrm{MeV}$ is influenced by the mass of the target however, with little problem evident in the light mass results as shown in Fig. 17. Given that those light mass nuclei as characterized as mostly "surface", we also believe that the problems with the effective interactions at the higher energies relates to the character of the force at central densities. 


\section{CONCLUSIONS}

A microscopic model of the nucleon-nucleus optical potential in coordinate space has been used to predict successfully the total reaction cross sections of nucleons from nuclei. That optical potential has been formed by folding complex energy- and density-dependent effective $N N$ interactions with OBDME of the target obtained primarily from shell models of the nuclei. As the approach accounts for the exchange terms in the scattering process, the resulting complex and energy dependent optical potential also is non-local. We have found that it is crucial to use effective $N N$ interactions which are based upon realistic free $N N$ interactions and which allow for modification from that free $N N$ scattering form due to nuclear medium effects of Pauli blocking and an average mean field. For optimum results, and for the light masses in particular, it is essential also to use the best (nucleon based) model specification of nuclear structure available. Marked improvement in results were obtained when, for ${ }^{9} \mathrm{Be}$ and ${ }^{12} \mathrm{C}$ in this study, complete $(0+2) \hbar \omega$ shell model calculations were used to define the OBDME required in the folding processes. For scattering from heavy nuclei at around $300 \mathrm{MeV}$, our results indicate the need for improvement in the effective $N N$ force possibly by explicit inclusion of $\Delta$ effects but also of those associated with the interaction at central field densities.

\section{Acknowledgments}

This research was supported by a research grant from the Australian Research Council, and also by DOE contract no. W-7-405-ENG-36.

[1] P. K. Deb, K. Amos, S. Karataglidis, M. B. Chadwick, and D. G. Madland, Phys. Rev. Lett. 86, 3248 (2001).

[2] W. Wlazlo et al., Phys. Rev. Lett. 84, 5736 (2000).

[3] X. Ledoux et al., Phys. Rev. Lett. 82, 4412 (1999).

[4] D. T. L. Jones, Acta Radiochim. 89, 235 (2001).

[5] B. A. Brown, Phys. Rev. Lett. 85, 5296 (2000).

[6] C. J. Horowitz and J. Piekarewicz, Phys. Rev. C 64, 062802(R) (2001).

[7] S. Karataglidis, K. Amos, , B. A. Brown, and P. K. Deb, Phys. Rev. C (2002), accepted for publication.

[8] K. Amos, P. J. Dortmans, H. V. von Geramb, S. Karataglidis, and J. Raynal, Adv. in Nucl. Phys. 25, 275 (2000).

[9] J. Raynal, computer code dwba91, nea 1209/02 (1991).

[10] J. Raynal, computer program dwba98, nea 1209/05 (1998).

[11] R. Machleidt, K. Holinde, and C. Elster, Phys. Rep. 149, 1 (1987).

[12] D. C. Zheng, B. R.Barrett, J. P. Vary, W. C. Haxton, and C. L. Song, Phys. Rev. C 52, 2488 (1995).

[13] P. Navrátil and B. R. Barrett, Phys. Rev. C 57, 3119 (1998).

[14] S. Karataglidis, P. J. Dortmans, K. Amos, and C. Bennhold, Phys. Rev. C 61, 024319 (2000).

[15] A. Lagoyannis et al., Phys. Lett. B518, 27 (2001).

[16] A. de Vismes and P. Roussel-Chomaz, private communication. 
[17] S. Karataglidis, P. J. Dortmans, K. Amos, and R. de Swiniarski, Phys. Rev. C 52, 861 (1995).

[18] L. Corragio, A. Covello, A. Gargano, N. Itaco, and T. T. S. Kuo, J. Phys. G 27, 2351 (2001).

[19] A. Johansson, U. Svanberg, and O. Sundberg, Arkiv Fysik 19, 527 (1961).

[20] R. F. Carlson et al., Phys. Rev. C 12, 1167 (1975).

[21] G. P. Millburn, W. Birnbaum, W. E. Crandall, and L. Schecter, Phys. Rev. 95, 1268 (1954).

[22] B. D. Wilkins and G. Igo, Phys. Rev. 129, 2198 (1963).

[23] P. Kirkby and W. T. Link, Can. J. Phys. 44, 1847 (1966).

[24] P. U. Renberg, D. F. Measday, M. Pepin, P. Schwaller, B. Favier, and C. Richard-Serre, Nucl. Phys. A183, 81 (1972).

[25] D. G. Montague, R. K. Cole, M. Makino, and C. N. Waddell, Nucl. Phys. A199, 457 (1973).

[26] W. F. McGill et al., Phys. Rev. C 10, 2237 (1974).

[27] I. Slaus, D. J. Margaziotis, R. F. Carlson, W. T. H. van Oers, and J. R. Richardson, Phys. Rev. C 12, 1093 (1975).

[28] A. Ingemarsson et al., Nucl. Phys. A653, 341 (1999).

[29] J. M. Cassels and J. D. Lawson, Proc. Phys. Soc. (London) A67, 125 (1954).

[30] E. J. Burge, Nucl. Phys. 13, 511 (1959).

[31] T. J. Gooding, Nucl. Phys. 12, 241 (1959).

[32] V. Meyer, R. M. Eisberg, and R. F. Carlson, Phys. Rev. 117, 1334 (1960).

[33] R. Goloskie and K. Strauch, Nucl. Phys. 29, 474 (1962).

[34] R. A. Giles and E. J. Burge, Nucl. Phys. 50, 327 (1954).

[35] M. Q. Makino, C. N. Waddell, and R. M. Eisberg, Nucl. Phys. 50, 145 (1964).

[36] J. J. H. Menet, E. E. Gross, J. J. Malanify, and A. Zucker, Phys. Rev. C 4, 1114 (1971).

[37] R. Chapman and A. M. Macleod, Nucl. Phys. A94, 313 (1967).

[38] V. Meyer and N. M. Hintz, Phys. Rev. Lett. 5, 207 (1960).

[39] K. Bearpark, W. R. Graham, and G. Jones, Nucl.Phys. 73, 206 (1965).

[40] R. E. Pollock and G. Schrank, Phys. Rev. 140, B575 (1965).

[41] J. F. Turner, B. W. Ridley, P. E. Cavanagh, G. A. Gard, and A. G. Hardacre, Nucl. Phys. 58, 509 (1964).

[42] J. F. Dicello and G. Igo, Phys. Rev. C 2, 488 (1970).

[43] R. D. Albert and L. Hansen, Phys. Rev. Lett. 6, 13 (1961).

[44] M. Q. Makino, C. N. Waddell, R. M. Eisenberg, and J. Hestenes, Phys. Lett. 9, 178 (1964).

[45] J. F. Dicello, G. J. Igo, and M. L. Roush, Phys. Rev. 157, 1001 (1967).

[46] C. Hojvat and G. Jones, Nucl. Instrum. Methods 66, 13 (1968).

[47] R. F. Carlson, A. J. Cox, T. Eliyakut-Roshko, and W. T. H. van Oers, Can. J. Phys. 73, 512 (1995).

[48] N. E. Davison et al., Nucl. Phys. A290, 45 (1977).

[49] R. Abegg et al., Nucl. Phys. A324, 109 (1979).

[50] S. Karataglidis, B. A. Brown, K. Amos, and P. J. Dortmans, Phys. Rev. C 55, 2826 (1997).

[51] S. Cohen and D. Kurath, Nucl. Phys. 73, 1 (1965).

[52] R. F. Carlson, Atomic Data and Nuclear Data Tables 63, 93 (1996).

[53] W. P. Abfalterer, F. B. Bateman, F. S. Dietrich, R. W. Finlay, R. C. Haight, and G. L. Morgan, Phys. Rev. C 63, 044608 (2001).

[54] R. W. Finlay, W. P. Abfalterer, G. Fink, E. Montei, T. Adami, P. W. Lisowski, G. L. Morgan, and R. C. Haight, Phys. Rev. C 47, 237 (1993).

[55] E. K. Warburton and B. A. Brown, Phys. Rev. C 46, 923 (1992).

[56] H. de Vries, C. W. de Jager, and C. de Vries, At. Data Nucl. Data Tables 36, 495 (1987). 
[57] X. Ji and B. H. Wildenthal, Phys. Rev. C 40, 389 (1989).

[58] A. Funk, H. V. von Geramb, and K. Amos, Phys. Rev. C 64, 054003 (2001). 